Wave Propagation in Jointed Geologic Media

T. Antoun

December 18, 2009 
This document was prepared as an account of work sponsored by an agency of the United States government. Neither the United States government nor Lawrence Livermore National Security, LLC, nor any of their employees makes any warranty, expressed or implied, or assumes any legal liability or responsibility for the accuracy, completeness, or usefulness of any information, apparatus, product, or process disclosed, or represents that its use would not infringe privately owned rights. Reference herein to any specific commercial product, process, or service by trade name, trademark, manufacturer, or otherwise does not necessarily constitute or imply its endorsement, recommendation, or favoring by the United States government or Lawrence Livermore National Security, LLC. The views and opinions of authors expressed herein do not necessarily state or reflect those of the United States government or Lawrence Livermore National Security, LLC, and shall not be used for advertising or product endorsement purposes.

This work performed under the auspices of the U.S. Department of Energy by Lawrence Livermore National Laboratory under Contract DE-AC52-07NA27344. 


\title{
Wave Propagation in Jointed Geologic Media
}

\author{
Tarabay Antoun \\ Lawrence Livermore National Laboratory
}

\section{Introduction}

Predictive modeling capabilities for wave propagation in jointed geologic media remain a modern day scientific frontier. In part this is due to a lack of comprehensive understanding of the complex physical processes associated with the transient response of geologic material, and in part it is due to numerical challenges that prohibit accurate representation of the heterogeneities that influence the material response. Constitutive models whose properties are determined from laboratory experiments on intact samples have been shown to over-predict the free field environment in large scale field experiments. Current methodologies for deriving in situ properties from laboratory measured properties are based on empirical equations derived for static geomechanical applications involving loads of lower intensity and much longer durations than those encountered in applications of interest involving wave propagation. These methodologies are not validated for dynamic applications, and they do not account for anisotropic behavior stemming from directional effects associated with the orientation of joint sets in realistic geologies. Recent advances in modeling capabilities coupled with modern high performance computing platforms enable physics-based simulations of jointed geologic media with unprecedented details, offering a prospect for significant advances in the state of the art. This report provides a brief overview of these modern computational approaches, discusses their advantages and limitations, and attempts to formulate an integrated framework leading to the development of predictive modeling capabilities for wave propagation in jointed and fractured geologic materials.

\section{Constitutive behavior of geologic materials}

Continuum mechanics based constitutive models are currently the only practical models for use in large scale (field scale) simulations of the dynamic response of geologic materials. These models rely on fitting parameters to phenomenologically describe the dependence of processes like yielding, bulking, damage and porous compaction on the deformation history and the rate of load application. The material response in these models is generally correlated to laboratory measured properties like density, elastic sound speed, stress-strain data under a range of loading conditions, and measurements of the strength and failure envelopes. Models developed in this manner have been shown to over-predict the free field environment in large scale field experiments. This is largely due to the effects of joints and other heterogeneities that are present at the field scale, but not in pristine laboratory samples. This is illustrated in Figures 1 and 2. Figure 1 compares measured and simulated particle velocity histories at two different ranges away from an underground nuclear detonation (Antoun et al., 2001). As shown in the figure, the simulation results are in reasonably good agreement with the data. However, 
matching the data required modifications to the model parameters originally calibrated using the static laboratory data. Specifically, a scaling law was introduced to degrade the yield and strength surfaces as shown in Figure 2. Phenomenologically, this is in line with experimental data that show the strength of

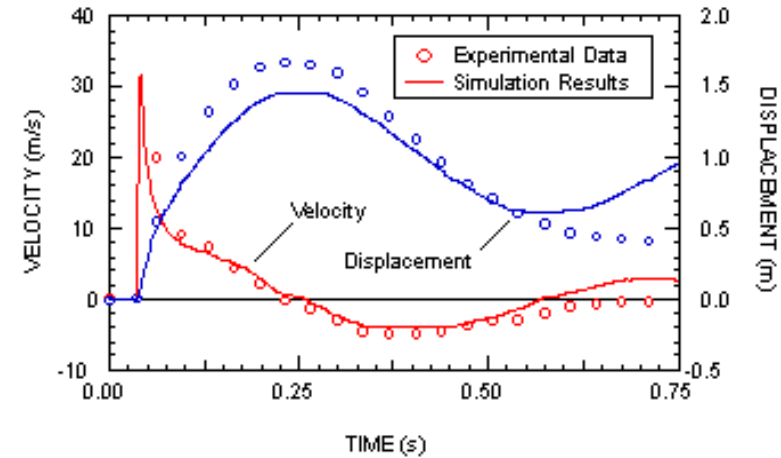

(a) slant range $=204 \mathrm{~m}$

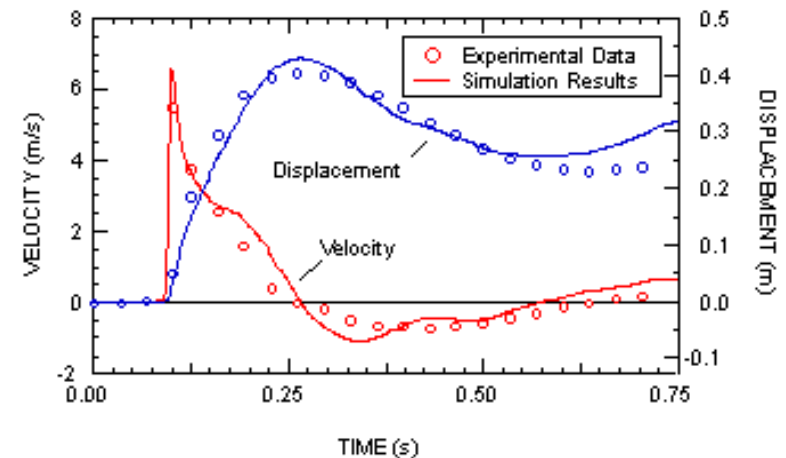

(b) slant range $=470 \mathrm{~m}$

Figure 1. Comparison of simulated and measured radial velocity and displacement histories at two different slant ranges away from the PILE DRIVER underground nuclear detonation.

granite and other geologic materials to be size-dependent, decreasing with increasing specimen dimensions (e.g. Hoek, 1994 and Hoek \& Brown, 1980). However, the manner in which this size scaling was introduced into the model was purely empirical. A function was added to the model to represent this effect, and its parameters were calibrated to achieve good agreement with the free field wave propagation data.

This modeling approach, whereby mechanistic data are used to calibrate the model, and the calibrated model is used to shed insight into the behavior of the geologic medium during wave propagation, is reasonable when sufficient data are available to calibrate the model. When calibrated in this manner, the applicability of the model is limited to the geologic medium for which it was calibrated. Extrapolation to other locations where the joint spacing and/or orientation may be different from the original location will require re-calibration of the constitutive model. This is

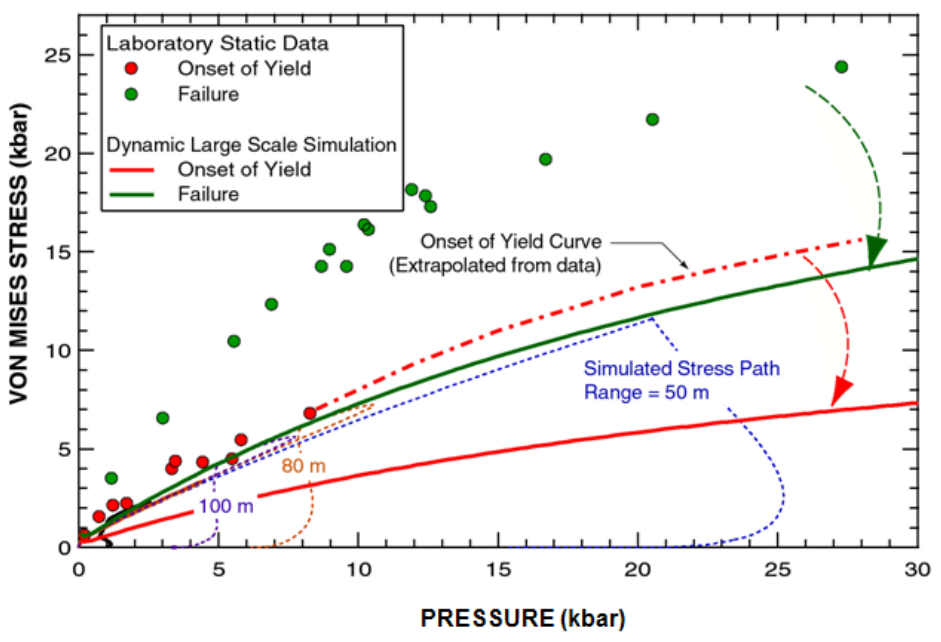

Figure 2. Yield and failure surfaces for granodiorite used in the PILE DRIVER calculations (solid curves) together with the data from static experiments (Schock et al., 1973). Dashed lines are the stress path trajectories experienced by the material at different ranges away from the source of explosion.

illustrated in Figure 3 which shows snapshots of the velocity field from two different 3D simulations of wave propagation in a jointed medium performed using LDEC (Heuze and Morris, 2007). The two snapshots on the left (Fig. 3a) 
correspond to a randomly jointed medium, while the snapshots on the right (Fig. 3b) correspond to a medium with bedding planes and regularized joints. Although the joint spacing and the contact properties used in the two calculations are the same, it is clear from Fig. 3 that joint orientation has a pronounced effect on the velocity distribution in the free field, and particularly in the vicinity of the tunnel. The constitutive model described above, which represents the state of the art in the groundshock modeling community, does not account for joint orientation and therefore is incapable of reproducing the kinds of effects shown in Fig. 3.
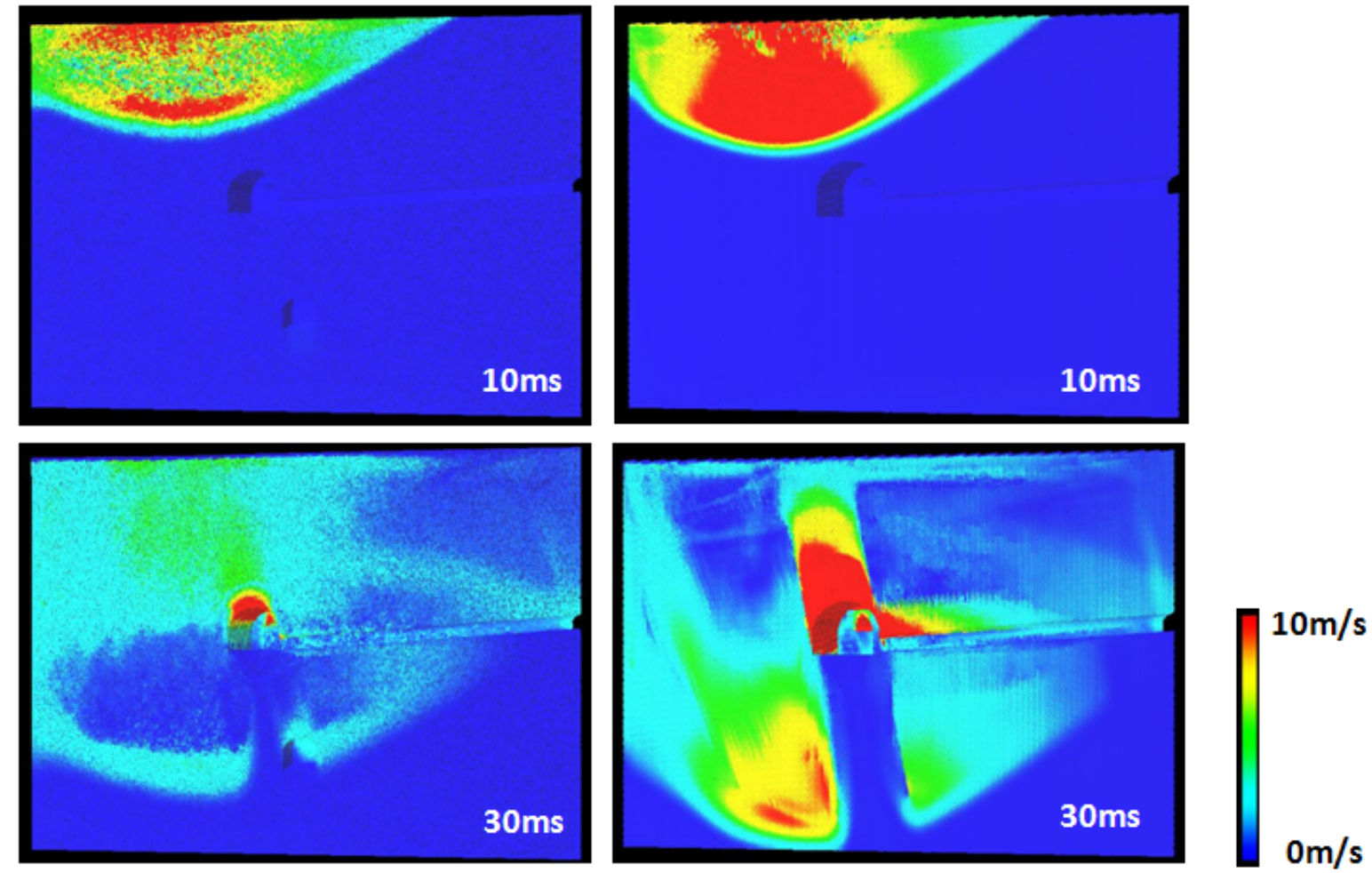

(a) Irregular (random) joints

(b) Regularized (bedded) geology

Figure 3. Snapshots of the velocity field from two different LDEC simulations of wave propagation in a jointed geologic medium (Heuze and Morris, 2007).

The currently-used modeling approach is best described as "descriptive", and is distinguished from the sought-after "predictive" modeling approach wherein the effects of joints and other heterogeneities are taken into account using a rational physics based approach that relates the macroscopic continuum response of the medium to elemental properties like joint spacing, joint orientation, and interface properties. One of the main objectives of this session on wave propagation in jointed media is to explore various modern numerical strategies that can be used to enable this predictive modeling capability.

\section{Equivalent Continuum Modeling of Jointed Media}

Lagrangian methods with conforming meshes and explicit inclusion of joints in the geologic model are well suited for analyzing wave propagation through geologic media. Unfortunately, current meshing 
tools are unable to automatically generate adequate hexahedral meshes for large numbers of irregular polyhedra, rendering it extremely difficult to develop accurate computational models for realistic geologies. Another concern is that joint stiffness in such explicit computations requires significantly reduced time steps, with negative implications for both the efficiency and quality of the numerical solution. These considerations hinder our ability to perform large scale simulations of wave propagation at the field scale using this explicit modeling approach. However, this approach is useful to perform simulations at the small scale using a representative geology containing $\sim 10^{5}$ blocks where the medium response to a variety of loading conditions can be assessed, and the knowledge gained from these detailed simulations can be encoded in a continuum model that can be used in large scale simulations.

This approach is illustrated in Figure 4 where the ratio of in situ strength to intact strength is plotted as a function of joint spacing. The figure shows significant strength degradation with increased joint spacing.

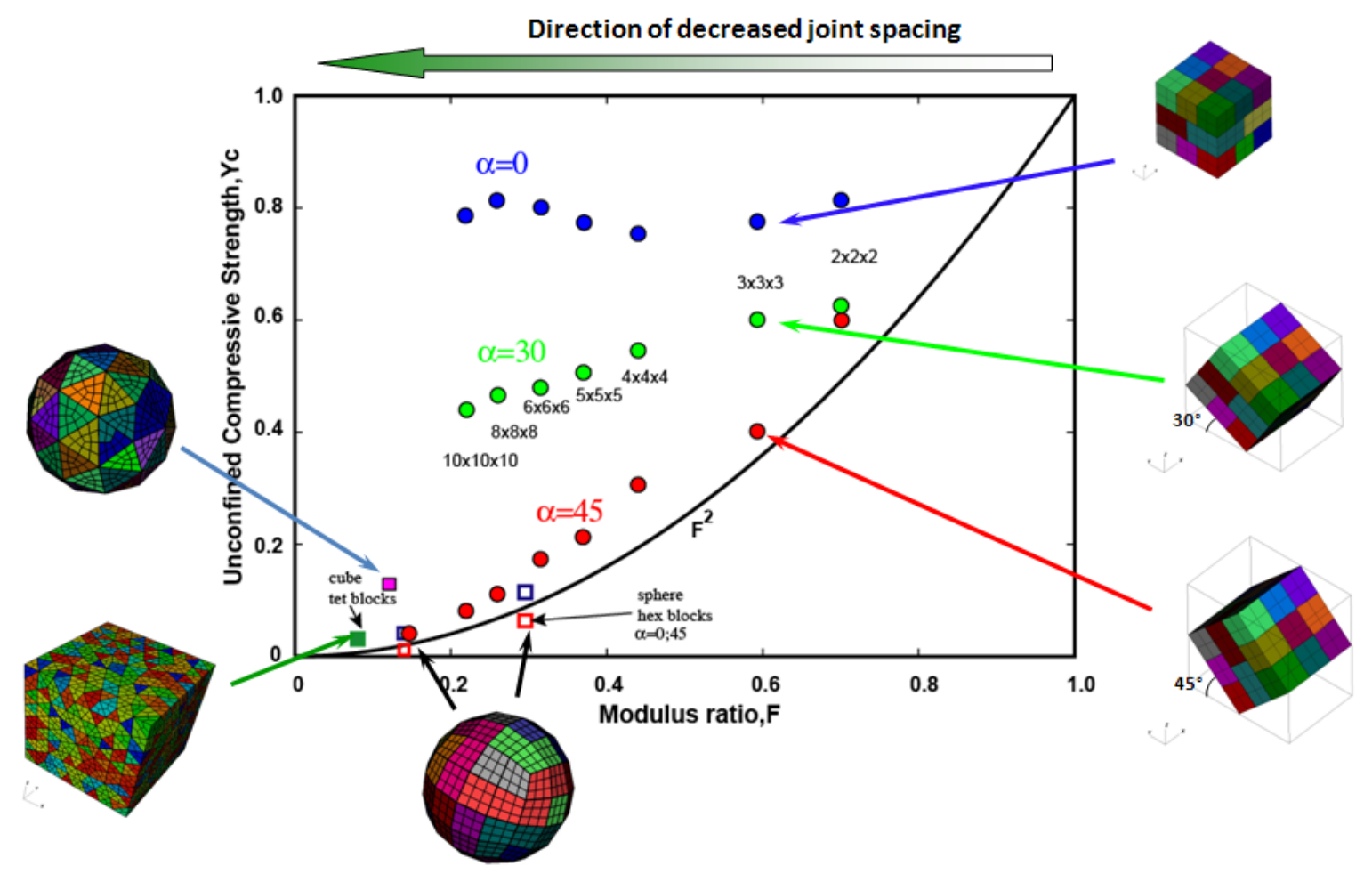

Figure 4. Effect of joints on the unconfined compressive strength of rock. The ratio of in situ strength to intact strength is plotted on the vertical axis and shows significant strength degradation as a function of increased joint spacing.

The figure also shows significant dependence of the in situ strength on joint orientation relative to the direction of load application. As a result, the emergent response of the jointed medium is generally anisotropic. Therefore, constitutive models for jointed media that properly account for joints and contact properties must be anisotropic. Such models are not currently being used, and the use of mesoscale simulations of the type described here to enable their development will be the focus of the workshop presentation and accompanying report by O. Vorobiev (2009). 


\section{Explicit Modeling of Joints in Eulerian Wave Propagation Calculations}

The use of non-conforming meshes in an Eulerian solver can alleviate many of the difficulties that render Lagrangian methods unpractical for large scale simulations of wave propagation in jointed media. With this approach, joints are treated as slip surfaces embedded in regular computational cells. The primary objective of this approach is to account for the effect of joints on wave profiles. The approach implicitly assumes that the joint thickness is always smaller then the cell size and uses a level set method to track the fault surface. Within each cell that contains an interface, directional joint plasticity is applied

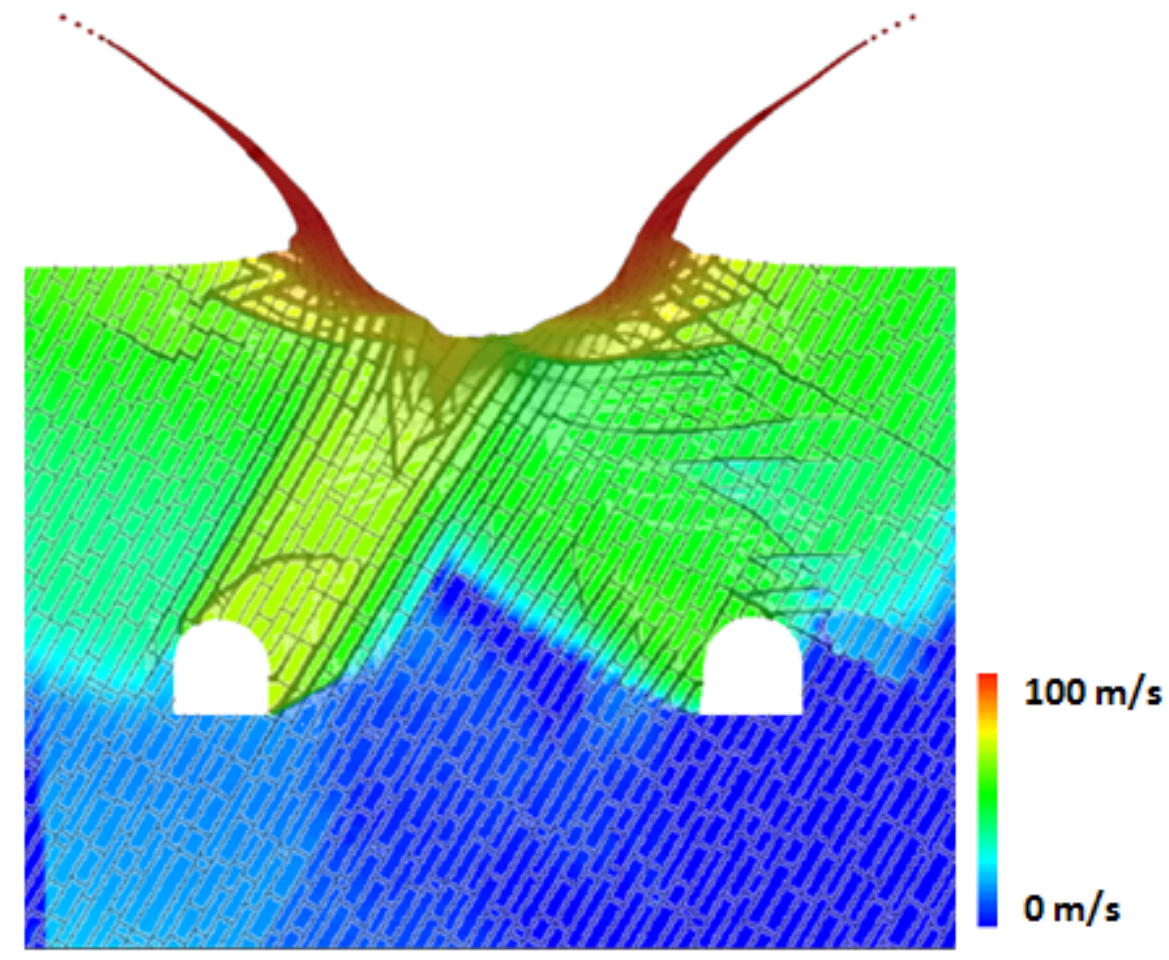

Figure 5. Simulation of wave propagation in a jointed rock mass. The figure illustrates the use of level sets to represent joints in Eulerian wave propagation calculations (Lomov e. al., 2009).

after the regular update of material state. One important advantage of the level set treatment is that the fault does not "diffuse" numerically during advection. This approach has been successfully demonstrated in serial 2D simulations (see Figure 5), and can be generalized to massively parallel 3D simulations. This modeling approach will be presented at the workshop by llya Lomov (Lomov et al., 2009) where it will be shown to be in good agreement with explicit Lagrangian methods for wave propagation problems. One limitation of this method is that it is not suitable for calculating discrete blocks and rubble motion where large joint displacements (larger than the cell size) may violate the inherent assumptions of the level set modeling approach.

\section{Explicit Modeling of Faults in Wave Propagation Calculations}

It was pointed out in the previous section that explicit representation of joints in large scale simulations of wave propagation in jointed media is prohibitively expensive. However, where it comes to modeling wave propagation across faults, explicit representation of the fault can be used, and it affords the most 
physically realistic approach for modeling the discontinuity. Faults can be represented explicitly in both Lagrangian and Eulerian frameworks. If the fault is of finite thickness, the thickness of the fault can be resolved in the simulation, and an appropriate constitutive model can be used to describe the response of the fault material. This is particularly convenient with codes that utilize adaptive mesh refinement, thereby making it possible to resolve regions of interest to centimeter-size zones when calculating problems with dimensions of hundreds of meters. This modeling approach was used by Lomov et al. 2003) to represent faults in a large scale simulation of the BANEBERRY underground nuclear detonation. The computational domain was a one cubic kilometer and the faults cut through the mesh as shown in Figure 6. This modeling approach will be discussed at the workshop by llya Lomov (Lomov et al., 2009).

The approach described above for representing joints in an Eulerian framework can also be used in Lagrangian codes. In this case, the mesh used to represent the fault can be conforming, or non-conforming, and the fault is discretized into finite elements with material properties representing the behavior of the fault. An alternate approach is to assume that the fault thickness is negligible and track the response of the fault using Lagrangian sliding surfaces. This approach will be described at the workshop by John

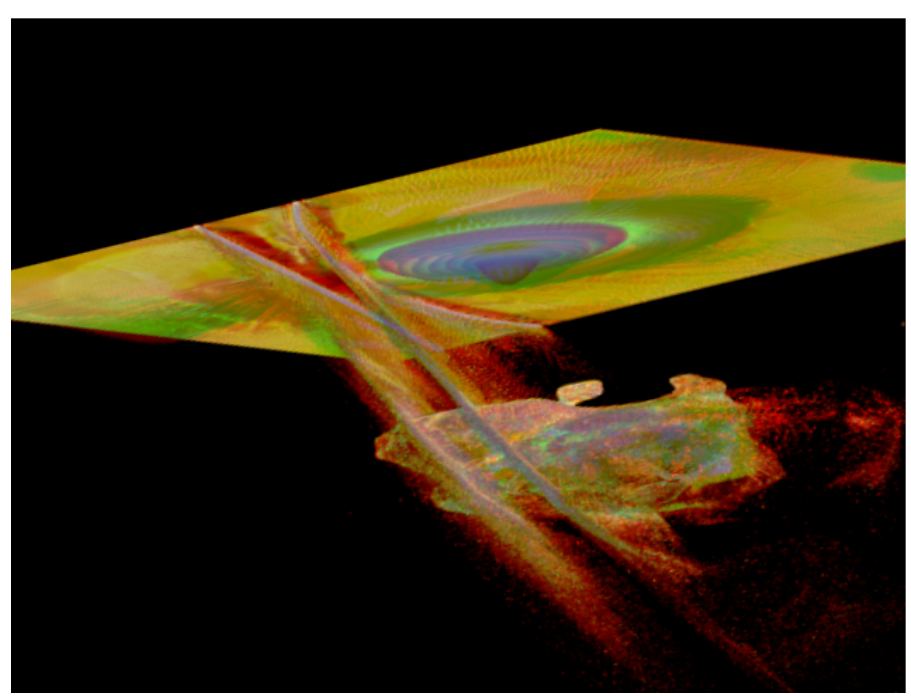

Figure 6. Damage field from a GEODYN simulation of the BANEBERRY underground nuclear detonation. Damage along the two faults that intersect the working point region is evident(Lomov et al., 2003).

Mould who investigated the effects of numerical approximations on fault modeling. The approximations included a smeared element approach where the fault is represented as a finite thickness zone of elements with properties selected to approximate the fault. Comparisons are made between aligning the grid with the fault and "stair-stepping" the fault at an oblique angle through a Cartesian mesh. Solutions are also presented using a conforming mesh and slide line algorithm where the fault is represented as a zero thickness interface, and the results are compared to an available analytical solution of a plane wave impinging on a planar fault characterized by friction and dilation angles.

\section{Numerical Convergence}

Simulations of wave propagation in geologic materials are inherently complicated. They involve hybrid numerical methods, with highly nonlinear material models. The quality of the numerical solution obtained in these simulations can be influenced by many factors, including mesh resolution. This is particularly important for applications involving large scale simulations of 3D wave propagation at the field scale. These simulations tend to be excessively large, straining the ability of the modeler to resolve features of interest. For most applications of interest, wave propagation in geologic media is an 
important element in the analysis of lethality and vulnerability of underground structures. Damage to these structures is generally a function of both the amplitude and duration of loading, both of which can be significantly altered by numerical artifacts stemming from inadequate mesh resolution. A detailed 1D study has been performed to quantify the effects of mesh resolution on the numerical solution (Key and Simons, 2009). In this study, an extreme fidelity one-dimensional spherical solution is developed for the far-field. It is a solution in one dimension that is definitive with respect to the mesh resolution needed to produce a high-fidelity, far-field wave profile. Following the establishment of a reference solution, two items are addressed: (1) an appraisal of the effect a lower resolution mesh customarily used in threedimensional high-fidelity simulations has on a representative far field calculation, and (2) the minimal mesh resolution needed to preserve $90 \%$ of the features found in the reference result.

\section{References}

1. Antoun, T.H., Lomov, I.N., and Glenn, L.A. "Development and Application of a Strength and Damage Model for Rock under Dynamic Loading," Proceedings of the 38th U.S. Rock Mechanics Symposium, Rock Mechanics in the National Interest, Edited by D. Elsworth, J. Tinucci, and K. Heasley, A.A. Balkema Publishers, Lisse, The Netherlands, 369-374 (2001)

2. Heuze, F., and J. Morris, "Insights into Ground Shock in Jointed Rocks and the Response of Structures Therein," International Journal of Rock Mechanics \& Mining Sciences 44, 647-676 (2007).

3. Hoek, E., "Strength of Rock and Rock Masses, ISRM News Journal, 2(2), 4-16 (1994).

4. Hoek, E., and E. T. Brown, Underground Excavations in Rock, The Institution of Mining and Metallurgy, London (1980).

5. Key, S., and D. Simons, "Mesh Convergence Study: A 1-D Spherical Far-Field Fidelity Study," to appear in these proceedings (2009).

6. Lomov, I., T. Antoun, and O. Vorobiev, "Comparison of Joint Modeling Approaches Including Eulerian Sliding Interfaces," to appear in these proceedings (2009).

7. Lomov, I., Antoun, T., Wagoner, J., and Rambo, J., "Three Dimensional Simulation of the Baneberry Nuclear Event," 13th American Physical Society Topical Conference on Shock Compression of Condensed Matter, Portland, OR (July 20-25, 2003).

8. Schock, R. N., Heard, H. C., and Stephens, D. R., "Stress-Strain Behavior of a Granodiorite and Two Craywackes on Compression to 20 Kilobars," J. Geophys. Res., Vol.78(36), pp. 5922-5941 (1973).

9. Vorobiev, O., and T. Antoun, "Equivalent Continuum Modeling for Shock Wave Propagation in Jointed Media," to appear in these proceedings (2009). 
Predictive

- Discontinuities in geologic media include faults, joints and material interfaces

- Show a cartoon representation

- how does each of these influence wave propagation?

- material properties modifications (anisotropy),

- impedence missmatches

- Include PILE DRIVER results showing that free field motions cannot be reproduced with a model that uses lab-measured properties.

- DG1 results showing good agreement with data when in situ properties are used.

- Baneberry-effect of faults on wave propagation

- Material interfaces:

O bonded?

O Sliding?

- Impedence mismatch

- Fault:

- normal compliance?

- Friction?

- Finite thickness?

- Numerical considerations?

- Joints

- Bedded vs random orientation

- Discrete vs homogenized representation

O

- Multiscale modeling: along the same lines as the granular materials SI 